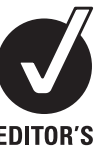

CHOICE

${ }^{1}$ Department of Paediatrics Prince Charles Hospital, Merthyr Tydfil, Glamorgan, UK ${ }^{2}$ Department of Paediatric Neurology, Bristol Royal Hospital for Children Bristol, UK

\section{Correspondence to}

Dr Andrew L Lux, Department of Paediatric Neurology, Bristol Royal Hospital for Children,

Floor 6, UHBristol Education Centre, Upper Maudlin Street, Bristol BS2 8AE, UK; andrew. lux@bristol.ac.uk

Accepted 30 July 2012

Published Online First

8 September 2012

\title{
Epilepsies in infancy
}

\author{
Shouja Alam, ${ }^{1}$ Andrew L Lux ${ }^{2}$
}

\section{ABSTRACT}

To evaluate and manage epileptic seizures and other paroxysmal events in infants, it is necessary to ask five key questions: (1) Is this a type of epilepsy?; (2) What seizure type(s) are occurring?; (3) Do these seizure types, combined with factors such as age at onset and EEG features, constitute an 'epilepsy syndrome'?; (4) What investigations do we need to do in searching for an underlying aetiology? and finally, (5) What is the prognosis for neurological and developmental state in later life?

This review considers epilepsies that have an onset in infancy but after the perinatal period, outlines the commoner epilepsy syndromes occurring in this age group and describes paroxysmal events that can mimic epilepsy. Epilepsies in infancy may be the manifestation of a genetic predisposition associated with a benign course and good prognosis for neurodevelopment. In contrast, they may pose the challenging situation of 'epileptic encephalopathy', rare but potentially treatable metabolic conditions, or structural abnormalities with poor developmental outlook and intractable seizures. Seizures in infancy are relatively rare and there is a wide range of underlying causes, some of which require specific treatments to avoid preventable neurodevelopmental damage. Guidance from the National Institute for Health and Clinical Excellence suggests early referral of cases of infantile epilepsy to a tertiary centre.

\section{INTRODUCTION}

In evaluating seizures and other paroxysmal events in infants, it is useful to ask the following five key questions: (1) Is this a type of epilepsy?; (2) What seizure type(s) are occurring?; (3) Do these seizure types, combined with factors such as age at onset and EEG features, constitute an 'epilepsy syndrome'?; (4) What investigations do we need to do in searching for an underlying aetiology? and finally, (5) What is the prognosis for neurological and developmental state in later life?

If we consider the first question to encompass a detailed and chronological description of the events (the 'phenomenology' or 'semiology'), and the final question to deal with behaviour, cognition and emotional and social functioning, then we can consider these five questions to have a useful correspondence with the five 'axes' proposed as a classification scheme by the International League Against Epilepsy (ILAE) in 2001. ${ }^{1}$

In this review, we consider epilepsies that have an onset in infancy but exclude seizures occurring in the perinatal and early neonatal period, which is a large subject in its own right. In particular, we outline paroxysmal events that mimic epilepsy and we describe the more common epilepsy syndromes occurring in this age group. And we suggest features that distinguish 'benign' from more severe epilepsy syndromes, these latter comprising a group that is often referred to as 'epileptic encephalopathies'. The focus here is on the recognition of key features rather than detailed management, but such recognition is vital for identifying appropriate interventions and predicting likely prognosis.

\section{QUESTION 1: ARE THE EVENTS LIKELY TO HAVE AN EPILEPTIC OR NON-EPILEPTIC BASIS?}

Even in specialist centres, there are high falsepositive rates for the diagnosis of epilepsy. This can result in missing other important diagnoses and exposing infants to unnecessary investigations and ineffective treatment interventions. Table 1 shows some of the commoner non-epileptic paroxysmal events that can mimic infantile epilepsies. We consider them in seven groups, although this is not a formal classification: (1) mainly rapid movements; (2) mainly sustained movements; (3) with eye movements as a prominent features; (4) complex movements and behaviours; (5) episodes with associated colour changes, loss of consciousness or weakness without a cardiac cause or (6) such episodes with a possible cardiac cause and (7) other metabolic, iatrogenic or induced illnesses.

In particular, care should be given to identifying potentially life-threatening conditions, such as longQT syndromes and tetralogy spells, or manifestations of serious conditions like severe gastro-oesophageal reflux (Sandifer syndrome) or neuroblastoma (opsoclonus-myoclonus syndrome). Diagnostic tips are shown in table 1.

Epilepsy should be distinguished from acute symptomatic seizures, which arise as the immediate result of a variety of acute insults, such as trauma, infection, hypoxia-ischaemia, hypoglycaemia, hypocalcaemia or other metabolic derangements. In contrast, epilepsy-perhaps better referred to as 'epilepsies' since their features and consequences are so varied-is considered to be unprovoked. (However, some epilepsies can be reflex, such as photic-stimulation-induced or reading epilepsies. In infants, reflex epilepsies are rare, but epileptic seizures can be caused by, for example, bathing in hot water.) If seizures recur when the original provoking factors have been removed, they are referred to as 'remote symptomatic epilepsies'. An example would be infantile spasms and focal seizures developing in a 6-month-old infant who had had a perinatal stroke.

Febrile seizures are the most common form of seizure in infancy. ${ }^{2}$ They occupy an ambiguous position but are probably best considered to be a form of acute symptomatic seizure rather than as a form of epilepsy, although this is arguable and there is an element of biological and genetic susceptibility. There is a large geographical variation that may be partly genetic and partly related 
Table 1 Some conditions that might mimic epilepsy in infancy

Condition Main features

\section{Mainly rapid movements}

Benign sleep myoclonus

Benign neonatal myoclonus or benign myoclonus of early infancy (BMEI)

Shuddering attacks

Jitteriness or tremulousness

\section{Mainly sustained movements}

Gastro-oesophageal reflux and Sandifer syndrome

Self-gratification disorder

Benign paroxysmal torticollis

Paroxysmal dyskinesia

Transient idiopathic dystonia of infancy

Hyperekplexia

\section{Eye movements a prominent feature}

Spasmus nutans

Paroxysmal tonic upgaze of infancy

Opsoclonus-myoclonus-ataxia syndrome

\section{Complex movements and behaviours}

Overflow movements

Stereotypies and self-stimulatory behaviours

Vitamin B12 deficiency

\section{Associated colour changes, loss of consciousness, or weakness WITHOUT a cardiac origin}

Breath-holding spells

Alternating hemiplegia of childhood

Paroxysmal extreme pain disorder

Reflex anoxic seizures refractory to treatment. clonazepam may help. seizure. paresis
Onset within a few weeks of age. Dramatic myoclonic movements confined to sleep occur in flurries, migrating from one limb then another in clusters of a few per second. Precipitated by gently rocking the sleeping infant. They do not wake nor distress the child. Settles within months.

Also referred to as 'benign non-epileptic infantile spasms', and with a recent suggestion that it be referred to as 'Fejerman syndrome'. This is associated with myoclonus, spasm-like movements, head nodding, blinking, and/or shuddering. The EEG is normal. Onset is usually between 1 and 12 months, and resolution by 3 years old. (Distinguish from 'benign myoclonic epilepsy of infancy' (see table 2)).

When presented with an interesting or new object/dinner, the child typically holds arms out and shudders involuntarily involving the whole body. Common variant of normal infant behaviour. Considered by some to be a variant of BMEI. Well recognised in the early neonatal period but can occur up to 6 weeks corrected age. The movements have symmetrical amplitude and speed in both directions, in contrast with clonus where one direction is faster. May be provoked by stimulus and settle with handling.

Gastro-oesophageal reflux in infants can cause tonic or repetitive truncal extension that may mimic seizures. It can also cause apnoea, which can also be mistaken for an autonomic seizure.

Older infants. Particularly in high chairs/car travel seats fitted with a strap between the legs AND if the child is bored. The insides of the thighs are rubbed together whilst legs are adducted in extension. Infant may be flushed and unresponsive. May be mistaken for tonic seizures or dystonia.

Recurrent episodes of cervical dystonia with typical onset in age range of 2-8 months. Apparent torticollis lasts hours to days and is associated with pallor and vomiting. There may be associated hearing impairment. Episodes usually stop by age 3 years but cases develop a tendency to migraine in later life.

Occur in infancy. Kinesiogenic (movement induced) or non-kinesiogenic.

Kinesiogenic — dystonia of unilateral leg. Often prodromal sensation. May have chorea or ballismus. Episodes last seconds to minutes, at a frequency of $100 \mathrm{~s}$ /day. Responds well to treatment.

Non-kinesiogenic - dystonia or choreoathetosis in face and limbs. Communication difficulties. Episodic ataxia. Lasts minutes-hours, at a frequency of $3 /$ day to $2 /$ year. Precipitated by fatigue, alcohol, caffeine and vibration. Tend to be

Described by Willemse. Onset before 6 months old. Dystonic posturing of an upper limb, typically with pronation and wrist hyperflexion, and sometimes posturing of the trunk and lower limb. The posturing usually resolves with intentional movements. Such dystonia has also been described in infants exposed in utero to cocaine.

Neonates markedly startle at sudden sounds or being touched (especially on the nose), resulting in severe total body stiffening. Life-threatening apnoea can result. Spells can be terminated by forcibly flexing the neck. Valproate and

A triad of asymmetrical ocular oscillations (pendular nystagmus), head nodding and torticollis or other unusual head positioning. Benign and self-limiting but need to rule out intracranial lesions.

A rare condition with onset typically before 10 months. Episodes of intermittent or sustained upward tonic gaze deviation that may last hours and downbeat nystagmus on downward gaze. Worse if fatigued or during an intercurrent illness. Should resolve by 3 years of age. Levodopa may help symptom control.

Opsoclonus refers to rapid, conjugate, irregular eye movements that are usually distressing for the child. It is often associated with myoclonus and ataxia as part of a paraneoplastic phenomenon associated with neuroblastoma.

These movements, such as 'mirror movements' of an upper limb, are involuntary and may rarely be mistaken for a focal

Stereotypies are repetitive, patterned and rhythmic movements that are considered to be involuntary, non-reflex and not goal-directed. Common forms are hand flapping, and lower limb shaking. They are often associated with excitement, boredom or stress. Tics tend to have an older age of onset. Stereotypical hand movements can occur in conditions with increased risk of epilepsy, such as Rett syndrome.

A movement disorder including mild choreoathetoid movements and/or a limb tremor resembling epilepsia partialis continua has been described in association with cobalamin deficiency.

Now often called 'reflex end expiratory apnoeic syncope' or 'prolonged expiratory syncope'. These can take cyanotic or pallid forms, both associated with vagal reflexes, bradycardia and decreased blood flow. Cyanotic episodes are usually provoked by anger or emotional upset, and are associated with apnoea in the expiratory phase of the breathing cycle. Pallid forms, usually referred to as 'reflex anoxic seizures' are discussed below.

The typical prodrome is restlessness and screaming. There can also be nystagmus (often unilateral) and episodes of dystonia or stiffening. Subsequent lateralised weakness can last minutes to days and can be mistaken for a Todd's

This condition, formerly known as 'familial rectal pain syndrome', is associated with mutations in nociceptive and sympathetic neuronal sodium channels. Clinical features may include startling, screaming, skin flushing (including the 'harlequin baby' appearance), pallor, apnoea, hypersalivation and generalised tonic stiffening lasting up to minutes. These 'pallid' episodes with altered consciousness may be reflex in nature but it is important to consider cardiac causes such as long-OT syndromes in addition to syncopal mechanisms, the common features being due to temporary inadequacy of cerebral blood flow. Reflex anoxic seizures are usually precipitated by surprise or minor trauma, and are more likely to have associated limb clonus. 
Table 1 Continued

\begin{tabular}{|c|c|}
\hline Condition & Main features \\
\hline \multicolumn{2}{|c|}{ Associated colour changes, loss of consciousness, or weakness WITH a possible cardiac origin } \\
\hline ALTEs and long-QT syndromes & $\begin{array}{l}\text { Apparent life-threatening events (ALTEs), some of which are likely to be manifestations of cardiac long-0T syndromes, } \\
\text { may be mistaken for primarily neurological events. Where loss of consciousness is a prominent feature, particularly } \\
\text { where it is associated with exercise, ensure that an ECG is performed. Long OT2 is associated with events provoked by } \\
\text { emotional or auditory stimuli, and long OT3 with events at rest and possibly in sleep. }\end{array}$ \\
\hline Tetralogy spells & $\begin{array}{l}\text { Episodes of cyanosis, dyspnoea and loss of consciousness associated with sudden right-to-left cardiac shunts. There } \\
\text { may be associated secondary seizures due to hypoxaemia. }\end{array}$ \\
\hline \multicolumn{2}{|c|}{ Other metabolic, iatrogenic or induced illnesses } \\
\hline Metabolic disturbances & $\begin{array}{l}\text { Hypoglycaemia and hypocalcaemia, often in association with other metabolic disturbances, can cause altered } \\
\text { consciousness or symptomatic seizures. }\end{array}$ \\
\hline Midazolam withdrawal syndrome & $\begin{array}{l}\text { This is commonly seen on paediatric intensive care units, and seems to be commoner where midazolam is combined } \\
\text { with fentanyl. Features may include altered awareness, agitation, vomiting, tremors and choreoathetoid or dystonic } \\
\text { movements. }\end{array}$ \\
\hline Fabricated or induced illness & $\begin{array}{l}\text { Infants are also susceptible to fabricated and induced illness, and it is prudent to keep this possibility in mind. It is } \\
\text { useful to have corroborative evidence from several observers about the nature of onset of the events. Presentations can } \\
\text { include suffocation or strangulation syncope, or administration of drugs that are detectable on urine toxicology screen. }\end{array}$ \\
\hline
\end{tabular}

to variations in exposure to the infectious agents precipitating the fever. On the island of Guam, for example, the reported cumulative incidence of febrile seizures in childhood is $14 \%$, in contrast with Western Europe and North America, where it is $<5 \%$.

Simple febrile seizures, as opposed to complex, are generalised (tonic-clonic in majority), short in duration ( $<15 \mathrm{~min}$ ) and do not recur within the same febrile illness. The risk of developing epilepsy is six times greater than the normal population (ie, $3 \%$, and the risk of developing epilepsy increases to $50 \%$ if all three of the following factors are present:

- abnormal neurodevelopmental status before the first episode;

- family history of epilepsy;

- complex febrile seizures.

It is important to avoid premature diagnostic closure in infants who have had previous febrile seizures since any seizure associated with fever might be due to meningoencephalitis, and febrile seizures can precede genuine epilepsy conditions, such as mesial temporal sclerosis and Dravet syndrome (see table 2). It is important to distinguish simple from complex febrile seizures, and to pay particular attention where febrile status epilepticus and/or prominent focal or lateralising features, either ictally or postictally, are present. It is also worth considering the family history in the context of the genetic/familial epilepsy syndromes such as generalised epilepsy with febrile seizures plus.

\section{QUESTION 2: WHAT SEIZURE TYPE(S) ARE OCCURRING?}

A broad range of seizures occur in infancy. The 2001 ILAE proposal suggests classifying seizures into two main groupsself-limited seizures and continuous seizures, the latter being the various forms of convulsive and non-convulsive status epilepticus. ${ }^{1}$ In either of these groups, seizures can be generalised or focal, which the proposed scheme describes in detail.

Routine interictal EEG has limited predictive value and should be used with caution as a means of identifying whether paroxysmal episodes are due to epilepsy or another paroxysmal condition. Some epileptiform abnormalities, such as central sharp waves, are common in conditions such as cerebral palsy even in the absence of witnessed seizures, and the absence of epileptiform discharges on an interictal EEG would never preclude epilepsy. On the other hand, EEG can help greatly to categorise seizures - the 'gold standard' being ictal video-EEG.
Some key points about seizure classification are:

- Home video may be invaluable and can be incomparably better than a poorly remembered event described verbally by a parent woken by a nocturnal event.

- Since even video-EEG can be difficult to interpret, surface electromyography, which shows the duration of muscle contraction, can help to distinguish seizure types. For example, myoclonus (duration up to $200 \mathrm{~ms}$ ) versus epileptic spasms (duration usually $1-2 \mathrm{~s}$ ) versus brief tonic seizures (several seconds in duration) versus atonic seizures or 'negative myoclonus' (absent surface EMG trace).

- Generalised seizures might be focal at onset and secondarily generalised, so avoid unjustifiable assumptions and be mindful of subtle focal seizure onset.

- Treatment choices are often made on the basis of seizure types rather than epilepsy syndromes or aetiology.

- Classification schemes evolve as insights and debate continue, and some terms are confusing or redundant. For example, the term 'astatic' indicates a fall from a standing position, which could be due to an atonic seizure (negative myoclonus), a violent myoclonic seizure or a brief tonic seizure. It is a good generic term for a 'drop attack' but a poor term for a seizure type.

\section{QUESTION 3: DOES THIS CONSTITUTE AN 'EPILEPSY SYNDROME'?}

An 'epilepsy syndrome' indicates a confluence of clinical features: typically a characteristic combination of age at onset, type or combination of seizures, and EEG pattern. The characteristic EEG pattern may be ictal and/or interictal. Identifying an infantile epilepsy syndrome: ${ }^{3}$

- helps in the choice of first-line treatment interventions;

- suggests a range of likely underlying causes;

- suggests a likely prognosis for seizure control and neurodevelopment;

- provides one form of inclusion criterion for clinical studies.

However, at least a quarter of the epilepsies that occur in infants do not easily fit into ILAE epilepsy syndrome classifications, and there is always a degree of inter-rater disagreement. ${ }^{4}$ Classification remains a dynamic process with the relationships between infantile epilepsy syndromes and aetiology being refined using video-EEG, improved brain imaging and new diagnostic techniques. Even seizure classification in infants can be particularly challenging. ${ }^{5}$ 
Table 2 Commoner epilepsy syndromes with onset in infancy

Epilepsy syndromes with generally good prognosis

Benign familial and non-familial infantile seizures

Benign familial neonatal seizures were described in 1964, with eight cases in three generations all developing seizures in the first few days of life but with good long-term prognosis. Similar benign seizures, both familial and non-familial, have been described with onset in later infancy, sometimes referred to as WatanabeVigevano syndrome. A number of known chromosomal loci or genetic mutations have been identified. ${ }^{6} 7$ Associations have been made with paroxysmal choreoathetosis that is variably expressed in these family members. $^{8} 9$

Benign familial neonatal-infantile seizures

Intermediate features to the conditions described above. Onset of seizures from 2 days to 6 months, and usually resolution by age 12 months. Some cases associated with mutations in the SCN2A gene (neuronal sodium channel, $\alpha-2$ subunit).

Benign infantile seizures associated with mild gastroenteritis

Not formally recognised as an epilepsy syndrome. Probably a situation-related or symptomatic seizure, and although seizures are often clustered and not associated with fever, later seizure recurrence is rare. Common in Japan and other parts of the Far East, and often associated with rotavirus infection.

Benign infantile focal epilepsy with midline spikes and waves during sleep Described by Capovilla and colleagues. ${ }^{9 a}$ Onset of seizures between 4 and 30 months, but usually between 12 and 18 months of age. A family history in half of cases. Activity arrest, vacant staring, cyanosis and automatisms. Vertex sharp waves are a normal feature of stage 2 sleep, but these EEG features are distinctive and epileptiform.

Benign myoclonic epilepsy of infancy

A rare condition that is commoner in male subjects. The myoclonic attacks have EEG correlates of spike-wave or polyspike-wave discharges - which distinguishes this from benign myoclonus of early infancy-but the interictal EEG is normal. The myoclonus tends to occur in the awake state but not during sleep.

Panayiotopoulos syndrome

Peak onset is at $4-5$ years old but cases have been described with onset as young as 1 year old. Prominent features are gaze deviation, impaired consciousness, prominent autonomic symptoms and ictal vomiting.

\section{Epilepsy syndromes that may be challenging to treat}

Early myoclonic encephalopathy

Ohtahara syndrome

Epilepsy of infancy with migrating focal seizures

Gelastic epilepsy associated with hypothalamic hamartoma

Dravet syndrome

West syndrome and infantile spasms

Doose syndrome of epilepsy with myoclonic-astatic seizures

Hemiconvulsion-hemiplegia-epilepsy (HHE) syndrome

Cryptogenic late-onset infantile spasms

Lennox-Gastaut syndrome

Myoclonic encephalopathy in non-progressive disorders

Progressive myoclonus epilepsies
This generally has onset in the neonatal period but can be up to 3 months of age. The myoclonias are often subtle and fragmentary, but there can be more prominent myoclonus, tonic spasms and focal seizures with eye deviation facial flushing and apnoea. EEG shows a suppression-burst pattern. Often associated with underlying metabolic disorders.

This generally has onset in the neonatal period but can be up to 3 months of age. Also known as 'early infantile epileptic encephalopathy'. Tonic seizures associated with suppression-burst pattern on EEG. A rare condition that may evolve into the commoner West syndrome.

Also known as migrating partial seizures of infancy. Onset usually before 6 months of age and after normal early development. Very frequent, multifocal seizures with onset from both hemispheres independently and often sequentially. These are generally intractable to treatment, though there are some case reports of response to intravenous levetiracetam. ${ }^{10}$ Developmental regression and a fall in the trajectory of head growth are usual.

Gelastic seizures involve short episodes of, often mirthless, laughter and other variable seizure symptoms. They are strongly association with hypothalamic hamartoma. Age of onset is variable but can be in infancy. Treatment is challenging but gamma-knife radiosurgery can be effective.

Onset generally from 3 months and $90 \%$ of cases with onset by 7 months of age. Seizures are provoked by intercurrent infection, immunisations or environmental factors that increase body temperature, such as hot baths. The seizures often increase in frequency and duration over time, and often have strongly lateralising features ('hemiconvulsive seizures'). Subsequent frequent myoclonus, other focal seizures, ataxia and developmental delay or regression. Variable EEG patterns and often photosensitivity. Many associated with mutations in SCN1A gene $(\alpha-1$ subunit of neuronal sodium channel). There is an increased risk of sudden unexpected death in epilepsy relative to most epilepsy conditions.

The commonest epilepsy syndrome in infancy. A combination of epileptic spasms with the interictal EEG pattern described as hypsarrhythmia is referred to as 'West syndrome'. The spasms are usually periodic with a frequency of between 5 and $30 \mathrm{~s}$, though sometimes outside this range. Although the truncal and limb flexion spasms are well known, clinical attacks may manifest with extensor limb and/or truncal semiology, or much more subtly with, for example, merely periodic eye deviation. Hypsarrhythmia is less likely in older infants with epileptic spasms, although the ictal EEG patterns are often the same.

Onset in late infancy or early childhood. 'Atonic' is probably a preferred term to 'astatic' since these are seizures with negative myoclonus. Myoclonus, falls, absences and generalised tonic-clonic seizures may all occur, and there is debate about how much the syndrome overlaps with Lennox-Gastaut and Dravet syndromes, though EEG features are generally distinguishable. Prognosis is variable and neurodevelopment good in some cases.

Peak onset incidence is up to 2 years of age but this seems to be a condition with declining incidence. Prolonged hemiconvulsion (lateralised convulsive status epilepticus) followed by flaccid hemiparesis, then progressive spasticity, and after a seizure-free period of months to several years, focal seizures with variable semiology. Suggested by Eisermann and colleagues as an epilepsy syndrome with features intermediate between West syndrome and Lennox-Gastaut syndrome. ${ }^{11}$

A combination of seizures - tonic, tonic-clonic, atypical absence and myoclonic-associated with the EEG pattern described as 'slow spike-wave' and paroxysmal fast activity in sleep. Onset in late infancy or early childhood. A less well-known syndrome with absence-type status epilepticus associated with myoclonus and ataxia. There is often a prominent startle response.

These generally have onset in later childhood, but it is worth being vigilant for cases of early-onset metabolic diseases such as neuronal ceroid lipofuscinoses. 
Table 2 outlines a number of epilepsy syndromes in infancy and their crude distinction into two categories: those with good prognosis and those who are likely to be challenging to treat.

\section{Challenging epilepsy syndromes: epileptic encephalopathies}

The concept of the 'epileptic encephalopathy' is that 'the epileptic activity itself may contribute to severe cognitive and behavioural impairment above and beyond what might be expected from the underlying pathology alone (eg, cortical malformation), and that this can worsen over time'. ${ }^{12}$ It is regarded as a 'pragmatic grouping' rather than a formal element of classification. The best-known examples are Ohtahara syndrome, West syndrome, and Lennox-Gastaut syndrome, but early myoclonic encephalopathy and Dravet syndrome are also generally considered to have the features of an epileptic encephalopathy. An implication is that the current and future cognitive and behavioural state can be modified by effective treatment aimed at controlling the epilepsy. This concept is supported by two retrospective studies of infantile spasms, where shorter lead times between symptom onset and treatment were shown to be associated with better long-term neurodevelopment. ${ }^{13} 14$

\section{QUESTION 4: CAN WE IDENTIFY AN UNDERLYING AETIOLOGY?}

Symptomatic epilepsy syndromes occur as a consequence of an identifiable primary cause such as a structural, neurodegenerative, metabolic, genetic or chromosomal disorder. An increasing number of genetic causes are being identified. ${ }^{15}$ West syndrome, the commonest epilepsy syndrome in infancy, is a good example of how epilepsy syndromes may have a variety of possible causes. Since optimal treatment and prognosis are strongly influenced by aetiology, it is best to consider this as a heterogeneous group of conditions sharing a non-specific phenotype: a series of infantile spasms. The diagnostic investigations suggested in figure 1 are adapted from a US expert consensus opinion as to how a clinician is recommended to approach an aetiological diagnosis. ${ }^{16}$ Although most cases of infantile spasms have identified underlying causes (and are referred to as having a 'symptomatic aetiology'), about 30\% are categorised as 'cryptogenic' or 'idiopathic'.

\section{Aetiological clues and investigations}

Seizures in infancy, at least after the neonatal period, have many potential causes and National Institute for Health and Clinical Excellence (NICE) guidance recommends early referral to an expert centre. There is no formulaic approach to investigating infantile epilepsy but there are some general principles. MRI is highly informative and is fundamental to the early assessment of aetiology. Subtler developmental abnormalities may be undetectable on early MRI scans because the process of brain myelination continues until about 30 months of age. Other clues include:

Symptoms and signs:

- Severe startle reactions and hiccups (often elicited from the prenatal history as occurring in utero) are associated

\begin{tabular}{|c|c|c|}
\hline $\begin{array}{l}\quad \text { History } \\
\text { HIE }^{a} \\
\text { Perinatal HIE } \\
\text { Near miss SIDS } \\
\text { Cardiac Arrest } \\
\text { Near Drowning } \\
\text { Maternal Toxaemia } \\
\text { Trauma } \\
\text { Encephalitis (usually Herpes) } \\
\text { Meningitis } \\
\text { Cerebral Abscess } \\
\text { Trans-placental Infections } \\
\text { Post-Cardiac Surgery } \\
\text { Neonatal Hypoglycaemia }\end{array}$ & $\begin{array}{l}\text { Neuroimaging/MRI } \\
\text { Tuberous Sclerosis } \\
\text { Aicardi Syndrome } \\
\text { Cortical Dysplasias } \\
\text { Lissencephaly } \\
\text { Pachygyria } \\
\text { Hemimegalencephaly } \\
\text { Band Heterotopia } \\
\text { Focal Cortical Dysplasia } \\
\text { Porencephaly } \\
\text { HIE } \\
\text { Tumour } \\
\text { AVM } \\
\text { Stroke } \\
\text { Leigh Disease } \\
\text { Hydranencephaly } \\
\text { Corpus Callosum Agenesis/ } \\
\text { Dysgenesis } \\
\text { Septo-Optico Dysplasia } \\
\text { Schizencephaly } \\
\text { Holoprosencephaly } \\
\text { Sturge-Weber Syndrome } \\
\text { Multiple Pineal Cysts } \\
\text { Trans-placental Infections } \\
\text { Peri-ventricular Leukomalacia } \\
\text { Krabbe Disease }\end{array}$ & 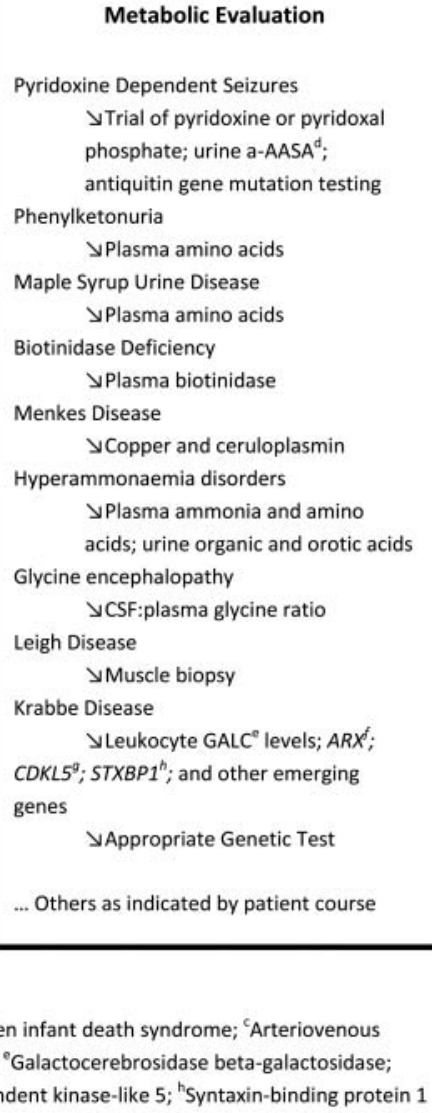 \\
\hline
\end{tabular}

Figure 1 Aetiological differential diagnosis for infantile spasms. Adapted from Pellock et al $2010 .{ }^{16}$ As yet, there is no standardised panel of genetic investigations. ${ }^{17}$ 
with glycine encephalopathy (non-ketotic hyperglycinaemia). This condition generally presents in the neonatal period with very severe seizures and a suppression-burst EEG (early myoclonic encephalopathy (see table 2)).

- Hypopigmented skin lesions, for which a Woods' lamp examination is required, are associated with tuberous sclerosis, which is strongly associated with focal seizures and with infantile spasms.

- Sparse, steely or 'kinky' hair suggests Menkes disease, which is investigated by microscopic analysis of hair and for low levels of plasma copper and caeruloplasmin (although the blood tests are insensitive in early infancy).

- Jitteriness and dystonic movements are often mistaken for epilepsy in infants presenting with glutaric aciduria type 1 (GA1). In GA1, there is usually macrocephaly and enlarged extra-axial cerebrospinal fluid (CSF) spaces, which can lead to a mistaken diagnosis of non-accidental head injury. In this and some other metabolic conditions, sodium valproate may worsen seizures by adverse effects on mitochondrial function, and treatment should include carnitine supplementation. ${ }^{18}$

- Faltering growth and hypotonia suggest mitochondrial cytopathies such as Leigh syndrome.

Biochemistry:

- Amongst the metabolic disorders that present with early myoclonic encephalopathy is molybdenum cofactor deficiency, which leads to functional deficit in the enzyme sulphite oxidase. Clues include low plasma uric acid and high urine xanthines and sulphites. Administration of cyclic pyranopterin monophosphate has recently been shown to be an effective treatment intervention, although it is not yet an approved treatment. ${ }^{19}$

- Congenital microcephaly, seizures and developmental delay may be due to disorders of serine synthesis, generally detected on plasma amino acids and amenable to prenatal and postnatal supplementation with serine and glycine.

- Biotinidase deficiency may mimic mitochondrial cytopathies, with developmental delay, episodes of encephalopathy and associated metabolic acidosis. It is usually associated with a severe rash, often with alopecia. Intractable epilepsy in infants is often treated with an empirical trial of biotin pending biotinidase assay results.

Electrophysiology:

- In early infancy, EEGs with burst-suppression patterns are more likely to have structural brain lesions where seizures are tonic in semiology (Ohtahara syndrome) and more likely to have a metabolic cause where seizures are predominantly myoclonic (early myoclonic encephalopathy) (table 2).

Genetics:

- Mutations in the STXBP1 (syntaxin-binding protein 1) gene affect neurotransmitter release and have been associated with Ohtahara syndrome and West syndrome. ${ }^{20}$

- A history of seizures and learning disability affecting solely female family members suggests a mutation in the PCDH19 (protocadherin) gene, which has X-linked inheritance. ${ }^{21}$

- Male infants with infantile spasms and/or myoclonic seizures may have mutations in the Aristaless-related homeobox gene. Other clinical features are variable and may include developmental delay, movement disorders, spasticity, abnormal genitalia and a spectrum of structural brain abnormalities that includes lissencephaly, midbrain malformations and agenesis of the corpus callosum. ${ }^{22}$
- Female infants who have a Rett-like phenotype with early-onset seizures (the 'Hanefeld variant') and acquired microcephaly may have mutations in the CDKL5 (cyclindependent, kinase-like) gene. ${ }^{23}$ The clinical and EEG features can be distinctive. ${ }^{24} \mathrm{~A}$ similar phenotype is found with mutations in the FOXG1 (forkhead box G1) gene. ${ }^{25}$

- Myoclonic encephalopathy may be associated with chromosomal disorders such as Angelman syndrome and chromosome $4 p$ deletions.

- Prolonged or hemi-convulsive seizures associated with fever should raise suspicions of abnormalities of neuronal sodium channel $\alpha-1$ subunits, which are confirmed by mutation analysis of the SCN1A gene. This gene is associated with a range of conditions, including Dravet syndrome (table 2) and what was formerly considered to be 'vaccine encephalopathy'. ${ }^{26}$

\section{METABOLIC CONDITIONS NOT TO BE MISSED Pyridoxine and pyridoxal phosphate}

Intractable seizures presenting in the neonatal period or early infancy are possibly due to rare but treatable metabolic diseases that should not be missed. These include pyridoxine-dependent epilepsy and pyridoxal phosphate-responsive epilepsy, which should be considered in any case of intractable epilepsy occurring under 2 years of age. ${ }^{27}$ Both conditions will respond to treatment with oral or nasogastric pyridoxal phosphate but this treatment is less well tolerated than oral pyridoxine-and neither is very well tolerated. The diagnosis should be considered as a differential when dealing with suspected hypoxic-ischaemic encephalopathy, which is relatively common, as the presentations can be similar.

- Pyridoxine-dependent epilepsy is due to deficiency of $\alpha$-AASA-dehydrogenase. Alpha-aminoadipic semialdehyde ( $\alpha$-AASA) is detected in blood and urine, with raised plasma pipecolic acid being a less sensitive marker. The biochemical abnormalities remain during treatment, so there is no reason for a trial of treatment to be delayed. Mutations of the antiquitin gene (ALDH7A1) confirm the genetic diagnosis. Seizures responsive to folinic acid are now considered to be the same condition.

- Pyridoxal phosphate-responsive epilepsy is due to deficiency of the enzyme pyridox(am)ine phosphate oxidase and genetic confirmation is by testing of the PNPO gene. Plasma amino acid testing may show raised glycine and threonine, as might CSF A more sensitive biochemical investigation is assay of CSF neurotransmitter metabolites, with low levels of pyridoxal-5'-phosphate levels, and possibly low levels of homovanillic acid and 5hyroxyindole acetic acid.

\section{Glucose transporter deficiency}

Glucose transporter-1 deficiency has become increasingly recognised and is important to bear in mind as the associated seizures are generally intractable to conventional antiepileptic drug treatment but ketogenic diet is usually effective (though less effective if movement disorders are prominent). A combination of seizures, movement disorder and faltering head growth should trigger consideration of the diagnosis, but the epilepsy phenotype is being recognised as increasingly broad. In infants, features may also include apnoea and cyanosis. In older infants and children, the features may be much more subtle. For example, it has been described in patients diagnosed as having benign myoclonic epilepsy of infancy, myoclonic-atonic 
('myoclonic-astatic') seizures or intractable absence-type seizures. $^{28}$

Glucose transporter-1 deficiency is detected by measuring absolute CSF glucose levels and the ratio of CSF glucose to immediate pre-lumbar puncture venous glucose (ie, before the stress of lumbar puncture); and by mutations in the SCL2A1 gene. Neither of these diagnostic approaches is $100 \%$ sensitive, so case definition can be challenging.

\section{Cerebral creatine deficiencies}

It has recently been recognised that epilepsy in infancy or early childhood can be due to cerebral creatine deficiency.

- Secondary to guanidinoacetate aminotransferase deficiency, this can be treated with creatine monohydrate and restriction of arginine. Other clinical features may include developmental delay, muscle hypotonia, extrapyramidal movements, abnormal eye movements and later, autistic and aggressive behaviour. Guanidinoacetate aminotransferase deficiency is detected by high levels of urine guanidinoacetate and absence of the normal creatine peak on magnetic resonance spectroscopy. An abnormal MRI signal in the globi pallidi is a less specific finding that has been reported in some cases.

- Cerebral creatine deficiency secondary to mutations in the cerebral creatine transporter gene (SLC6A8) on the $\mathrm{X}$-chromosome are not effectively treated with creatine supplementation. They seem to be associated with slightly later-onset epilepsy but the clinical phenotypes need fuller determination.

- Deficiency of arginine:glycine amidinotransferase appears to be the least common form of cerebral creatine deficiency, with the main features being developmental delay and autism rather than epilepsy.

\section{Delay, epilepsy and neonatal diabetes syndrome}

The combination of developmental delay, epilepsy and neonatal diabetes is found with mutations in the potassium-ATP channel Kir6.2. ${ }^{29}$ This can present as infantile spasms. Neurological outcome can be improved by early treatment with oral sulphonylurea, whereas insulin treatment is ineffective and will not protect development.

\section{TREATMENT OF INFANTILE EPILEPSY}

Clearly, the first steps to effective treatment are correctly recognising epilepsy, seizure types and underlying causes. Treatment with antiepileptic drugs should not inhibit an appropriate search for treatable metabolic causes. Treatments are not discussed in detail here but a few learning points are noted.

\section{Treatment guidelines}

Guidelines on the treatment of epilepsy have been produced by the UK NICE and by the Scottish Intercollegiate Guidelines Network. The most recent NICE guidance ${ }^{30}$ (CG137) states that the diagnosis and management of epilepsy within the first few years of life may be extremely challenging. For this reason, children with suspected epilepsy should be referred to tertiary services early, because of the profound developmental, behavioural and psychological effects that may be associated with continuing seizures'.

\section{Sodium valproate}

Sodium valproate is subject to national and cultural differences relating to perceived risks and use in infancy. It is a less commonly used first-line treatment in North America than in
Europe and can be problematic in some situations. For example, in GA1 and in combination with the ketogenic diet, it can deplete carnitine. It is also problematic in mitochondrial disorders, and in particular, Alpers disease and mutations in the POLG1 (polymerase gamma) gene, where it can interfere with mitochondrial metabolism and precipitate liver failure.

\section{Ketogenic diet}

A ketogenic diet can be an effective intervention where antiepileptic drugs have been ineffective. The diet is modified in infants in order to minimise risks to growth. Its strongest indications are glucose transporter deficiency and pyruvate dehydrogenase (E1) deficiency, where the metabolic defects preclude brain utilisation of carbohydrates. However, it is contraindicated in pyruvate carboxylase deficiency, porphyria and defects of fatty acid oxidation

\section{Epilepsy surgery}

Even in infancy, intractable epilepsy can be treated surgically. Indeed, early intervention is likely to reduce secondary epileptogenesis, and the maturing brain has a greater propensity for plasticity than the older brain, so there might be significant advantage associated with early referral and investigation.

\section{QUESTION 5: WHAT IS THE PROGNOSIS? Development and comorbidities}

ILAE axis 5 comprises comorbidities, the associated cognitive, behavioural, emotional and developmental features that may be associated with the epilepsy. This corresponds with an important question that will be asked by parents and families of infants with epilepsy: 'What is the prognosis for development?' One of the great challenges facing the paediatrician is to reliably identify any underlying cause, which will strongly predict prognosis for the development of comorbidities, but also to appreciate where extensive metabolic and genetic investigation is unlikely to yield such an aetiological diagnosis given the current state of knowledge in this field.

When counselling families about prognosis for development, it is prudent to bear in mind case ascertainment bias. Early reports of cases with recently identified conditions tend to come from teaching centres, which generally have a more severe case mix. There can be period effects, with less severe cases being identified over time because investigations are performed more frequently and phenotypic spectra are recognised as being broader than previously imagined. Furthermore, in providing information about prognosis, it is also prudent to consider the effects over time of more effective treatment interventions, even if merely symptomatic or supportive treatments. For newer genetic conditions, information about longerterm prognosis is likely to be limited.

It is important to keep in mind the underlying motivation behind treating a child with epilepsy. It might not always be possible to stop seizures, in which case it is necessary to find balance between reasonable seizure control and unacceptable adverse effects associated with the treatments themselves.

\section{Risks of sudden death?}

One controversial issue is that of counselling risks of sudden unexpected death in epilepsy. Although nobody wishes to increase stress in an already stressful situation, NICE guidance encourages healthcare professionals to share and contextualise this information. The families of people who have suffered sudden death generally state that they would have preferred that information to have been offered before the event. 


\section{Summary and key learning points}

Epilepsies in infancy may be the manifestation of a genetic predisposition associated with a benign course and good prognosis for neurodevelopment. However, they may pose a challenging situation of 'epileptic encephalopathy', rare but potentially treatable metabolic conditions, or structural abnormalities with poor developmental outlook and intractable seizures. The 2001 ILAE 5-axis proposal provides a pragmatic framework for clinical diagnosis and management, although newer classification schemes are being developed. However, seizures in infancy are relatively rare and there are a wide range of underlying causes, some of which require specific treatments to avoid preventable neurodevelopmental damage. NICE guidance recommends early referral of cases to a tertiary centre.

Mortality from sudden unexpected death in epilepsy or other causes is higher in infants with epilepsy than in other children. For example, conditions such as Ohtahara syndrome, migrating epilepsy of infancy and Dravet syndrome have significant excess mortality in spite of appropriate treatments.

\section{SOURCES OF INFORMATION AND TRAINING}

The websites of Epilepsy Action (http://www.epilepsy.org.uk/) and the Epilepsy Society (http://www.epilepsysociety.org.uk/) (formerly the National Society for Epilepsy) have very useful information for patients, families and professionals. Infantile seizures and epilepsies are covered during days 1 and 2 of the paediatric epilepsy training level 2 training course run by the British Paediatric Neurology Association.

Acknowledgements ALL is treasurer of the British Paediatric Neurology Association, a not-for-profit organisation that runs the paediatric epilepsy training courses mentioned in this article.

Competing interests None.

Provenance and peer review Commissioned; externally peer reviewed.

\section{REFERENCES}

1. Engel J. A proposed diagnostic scheme for people with epileptic seizures and with epilepsy: report of the ILAE task force on classification and terminology. Epilepsia 2001; $42: 796-803$

2. Lux AL. Treatment of febrile seizures: historical perspective, current opinions, and potential future directions. Brain Dev 2010;32:42-50.

3. Korff CM, Nordli DR. Epilepsy syndromes in infancy. Pediatr Neurol 2006;34:253-63.

4. Sarisjulis N, Gamboni B, Plouin $\mathrm{P}$, et al. Diagnosing idiopathic/cryptogenic epilepsy syndromes in infants. Arch Dis Child 2000;82:226-30.

5. Hsieh DT, Walker JM, Pearl PL. Infantile seizures: infants are not just little children. Curr Neurol Neurosci Rep 2008;8:139-44.

6. Fukuyama $\mathbf{Y}$, Arima $\mathrm{M}, 0 \mathrm{kada}$ R, et al. Electro-clinical characteristics of epilepsies in infancy and childhood. Paediatr Univ Tokyo 1963;59:11-6.

7. Vigevano $\mathbf{F}$, Specchio N, Caraballo $\mathrm{R}$, et al. Benign familial and nonfamilial seizures. In: J Engel J, Pedley TA, eds. Epilepsy: a Comprehensive Textbook. Philadelphia: Wolters Kluwer-Lippincott Williams \& Wilkins, 2008:2313-21.

8. Rochette J, Roll P, Szepetowski P. Genetics of infantile seizures with paroxysmal dyskinesia: the infantile convulsions and choreoathetosis (ICCA) and ICCA-related syndromes. J Med Genet 2008;45:773-9.
9. Szepetowski P, Rochette J, Berquin P, et al. Familial infantile convulsions and paroxysmal choreoathetosis: a new neurological syndrome linked to the pericentromeric region of human chromosome 16. Am J Hum Genet 1997;61:889-98.

9a. Capovilla G, Beccaria F, Montagnini A, et al. Benign focal epilepsy in infancy with vertex spikes and waves during sleep. Delineation of the syndrome and recalling as 'benign infantile focal epilepsy with midline spikes and waves during sleep' (BIMSE). Brain Dev 2006;28:85-91.

10. Cilio MR, Bianchi R, Balestri M, et al. Intravenous levetiracetam terminates refractory status epilepticus in two patients with migrating partial seizures in infancy. Epilepsy Res 2009;86:66-71.

11. Eisermann MM, Ville D, Soufflet C, et al. Cryptogenic late-onset epileptic spasms: an overlooked syndrome of early childhood? Epilespsia 2006; 47:1035-42.

12. Berg AT, Berkovic SF, Brodie MJ, et al. Revised terminology and concepts for organization of seizures and epilepsies: report of the ILAE commission on classification and terminology, 2005-2009. Epilepsia 2010;51:676-85.

13. Kivity S, Lerman P, Ariel R, et al. Long-term cognitive outcomes of a cohort of children with cryptogenic infantile spasms treated with high-dose adrenocorticotropic hormone. Epilepsia 2004;45:255-62.

14. Eisermann MM, DeLaRaillère $A$, Dellatolas $G$, et al. Infantile spasms in Down syndrome- effects of delayed anticonvulsive treatment. Epilepsy Res 2003;55:21-7.

15. Nabbout R, Dulac 0. Epileptic syndromes in infancy and childhood. Curr Opin Neurol 2008;21:161-6.

16. Pellock JM, Hrachovy R, Shinnar S, et al. Infantile spasms: a U.S. consensus report. Epilepsia 2010;51:2175-89.

17. Pal DK, Pong AW, Chung WK. Genetic evaluation and counseling for epilepsy. Nat Rev Neurol 2010;6:445-53.

18. Kölker S, Christensen E, Leonard JV, et al. Diagnosis and management of glutaric aciduria type L-revised recommendations. J Inherit Metab Dis 2011;34:677-94.

19. Veldman A, Santamaria-Araujo JA, Sollazzo S, et al. Successful treatment of molybdenum cofactor deficiency type A with cPMP. Pediatrics 2010:125 e1249-54.

20. Otsuka M, Oguni $\mathrm{H}$, Liang JS, et al. STXBP1 mutations cause not only Ohtahara syndrome but also West syndrome - result of Japanese Cohort Study. Epilepsia 2010;51:2449-52.

21. Dibbens LM, Tarpey PS, Hynes K, et al. X-Linked protocadherin 19 mutations cause female-limited epilepsy and cognitive impairment. Nat Genet 2008;40:776-81.

22. Suri M. 'The phenotypic spectrum of ARX mutations'. Dev Med Child Neurol 2005;47:133-7.

23. Pintaudi M, Baglietto MG, Gaggero R, et al. Clinical and electroencephalographic features in patients with CDKL5 mutations: two new Italian cases and review of the literature. Epilepsy Behav 2008;12:326-31.

24. Melani F, Mei D, Pisano T, et al. CDKL5 gene-related epileptic encephalopathy: electroclinical findings in the first year of life. Dev Med Child Neurol 2011:53:354-60

25. Ariani F, Hayek G, Rondinella D, et al. FOXG1 is responsible for the congenital variant of Rett syndrome. Am J Hum Genet 2008;83:89-93.

26. Berkovic SF, Harkin L, McMahon JM, et al. De-novo mutations of the sodium channel gene SCN1A in alleged vaccine encephalopathy: a retrospective study. Lancet Neurol 2006:5:488-92.

27. Stockler S, Plecko B, Gospe SM, et al. Pyridoxine dependent epilepsy and antiquitin deficiency: clinical and molecular characteristics and recommendations for diagnosis, treatment and follow-up. Mol Genet Metab 2011;104:48-60.

28. Gaspard N, Suls A, Vilain C, et al. 'Benign' myoclonic epilepsy of infancy as the initial presentation of glucose transporter-1 deficiency. Epileptic Disord 2011:13:300-3.

29. Bahi-Buisson N, Eisermann M, Nivot $\mathrm{S}$, et al. Infantile spasms as an epileptic feature of DEND syndrome associated with an activating mutation in the potassium adenosine triphosphate (ATP) channel, Kir6.2' . J Child Neurol 2007;22:1147-50.

30. National Institute for Clinical Excellence (NICE). 2012. 'The epilepsies: the diagnosis and management of the epilepsies in adults and children in primary and secondary care', January 2012, p. 41, www.nice.org.uk/nicemedia/live/13635/ 57779/57779.pdf (accessed 1 May 2012). 